\section{Estimación del tiempo de consulta ambulatoria en clínica médica}

\author{
DELIA OUTOMURO ${ }^{1,2}$, ANDREA MARIEL ACTIS $2,3, \mathrm{a}$
}

\section{Analysis of ambulatory consultation length in medical clinics}

\begin{abstract}
Planning a schedule for medical appointments in health services must be efficient and flexible, but also it has to meet the needs of patients, health professionals and nonmedical staff. There are large differences in the opinion about the optimal duration to meet these objectives, across countries. In this paper we propose to perform a review of the literature to estimate the appropriate length of a medical consultation in primary care, based on international standards. We conclude that managers of health systems should rethink the way they organize the agenda for medical appointments. Medical and bioethical reasons suggest assigning a lapse close to 20 minutes for consultations in medical clinics.
\end{abstract}

(Rev Med Chile 2013; 141: 361-366).

Key words: Delivery of health care; Health care surveys; Health Services research.

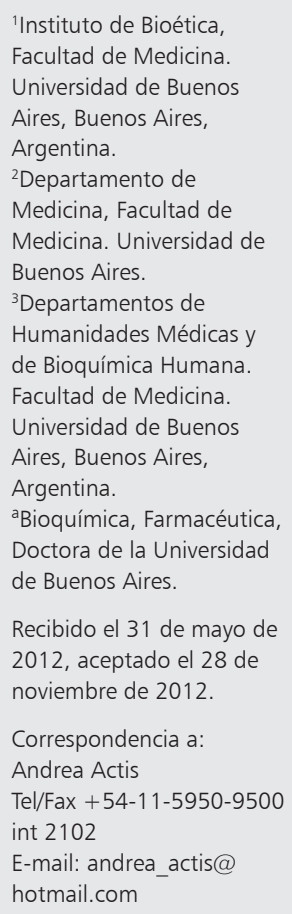

$\mathrm{P}$ lanificar la organización de un sistema de consultas médicas ambulatorias (CMA) no es un tema sencillo, pues requiere tener en cuenta una serie de cuestiones que resultan cruciales para la toma de decisiones. Establecer el número de CMA por día y por semana implica conocer, por un lado la disponibilidad de recursos humanos y físicos y, por otro, la demanda de turnos por parte de la población. Estos datos resultarán de la interacción con el área administrativa pertinente que, año a año, irá poniendo al día las estadísticas según cada especialidad ${ }^{1}$.

La construcción de un sistema de turnos debe ser eficiente y flexible pero también debe funcionar en beneficio tanto de pacientes como de los profesionales de la salud y del personal no médico ${ }^{2}$. En nuestro medio (Buenos Aires, Argentina) observamos que el tiempo es, en promedio, de quince minutos en clínica médica, pudiendo ser algo mayor en algunas especialidades como pediatría o salud mental.

La decisión acerca de cuánto tiempo el profesional de la salud estará cara a cara con el paciente resulta vital no sólo para programar la agenda de turnos sino también para garantizar la calidad de atención médica. Un turno demasiado corto ha sido descripto, entre otras cosas, como uno de los factores que impide la realización de una buena promoción de la salud. La importancia de la promoción de la salud ha sido enfatizada a nivel internacional desde la OMS y esto incluye todos los aspectos relacionados con aquellas actividades que buscan mejorar el nivel de salud de los individuos y de la comunidad. Discutir estilos de vida y solicitar estudios complementarios es lo que más hace un clínico en sus consultas de rutina, fundamentalmente en consultas de primera vez $z^{3}$.

Existen grandes diferencias según los países acerca de cuál es la duración óptima para cumplir con estos objetivos.

En este trabajo nos proponemos realizar una revisión bibliográfica de los estándares a nivel internacional para establecer el tiempo de CMA que resulte éticamente aceptable en clínica médica. Cabe destacar que la cantidad de bibliografía hallada sobre este tema no ha sido muy voluminosa a pesar de que la preocupación de los profesionales sobre el tema indica que debería dedicársele mayor atención a este tema ${ }^{4,5}$. 


\section{La situación a nivel mundial}

Durante la década 1990-99 muchos sistemas pasaron de tener CMA de 7,5 min a $10 \mathrm{~min}$ en el área de clínica médica y este cambio redundó en el mutuo beneficio tanto de pacientes, que sentían un mejor trato y calidad de atención, como también de los médicos que disminuyeron su nivel de estrés, observándose una disminución del síndrome profesional de burn out ${ }^{3}$. En otro estudio, se observó que al incrementar de 7,5 a 10 min la duración de la CMA mejoraba notablemente la promoción de la salud, sobre todo a nivel de la detección de hipertensión arterial; de esta manera se demostraba que el tiempo dedicado a la toma de la tensión arterial es un factor importante en la duración total de la consulta. También se observaron mejoras en la detección de tabaquismo y alcoholismo, indicando que una mayor duración de la CMA puede contribuir a mejorar la calidad de vida de los pacientes en el largo plazo, al permitir detectar factores de riesgos para la salud ${ }^{6}$.

En España existe una amplia disparidad en los tiempos asignados, considerándose $20 \mathrm{~min}$ en Zaragoza y 10 min en Madrid. En Rusia, el tiempo de consulta está reglamentado en $10 \mathrm{~min}$, los que deben distribuirse de la siguiente manera: $1 \mathrm{~min}$ para saludar y despedir al paciente, 3 min para la anamnesis, 2 min para el examen físico, $2 \mathrm{~min}$ para la prescripción y 2 min para cumplimentar formalidades administrativas. En El Salvador, el médico general cuenta con turnos de $10 \mathrm{~min}$, en tanto que en Perú, 12 minutos ${ }^{7}$.

En los Estados Unidos de Norteamérica, un estudio que recopiló información de más de 46.000 consultas médicas entre los años 1997 y 2005, demostró que el tiempo promedio de consulta había aumentado de 16 a 20,8 min, registrándose un incremento de 3,4 min para las consultas con el médico general ${ }^{8}$.

En Japón los pacientes utilizan una frase que describe, irónica pero dramáticamente, las condiciones de la atención médica en ese país: los japoneses dicen "esperar tres horas para que nos atiendan en 3 min". Otro estudio revela que el promedio de duración de las consultas es de 6 min $^{9}$.

Una investigación en Alejandría, Egipto, mostró que los tiempos de consulta oscilan entre $14,5 \pm 9$ min para una consulta de primera vez y entre 12,3 $\pm 3,9$ min en visitas posteriores ${ }^{10}$. En Turquía, un estudio patrocinado por la Organi- zación Mundial de la Salud (OMS) reveló que el tiempo promedio de la consulta médica era de $11 \mathrm{~min}^{11}$. En Etiopía, otra investigación puso de manifiesto que la consulta es actualmente de unos $6,26 \pm 2,55 \mathrm{~min}$, pero los pacientes esperan que la misma dure unos $14,02 \pm 6,73$ para considerarse bien atendidos ${ }^{12}$.

En Canadá hay básicamente dos sistemas de salud: uno donde el médico cobra honorarios por cada consulta y otro en el cual tiene un salario mensual. Los tiempos de consulta en el primer caso varían entre 10 y 15 min mientras que en el segundo, lo hacen entre 20 y $45 \mathrm{~min}^{13}$.

Finalmente, en la Argentina existe escasa bibliografía al respecto; sin embargo, es de destacar un estudio donde se pone de manifiesto la preocupación de los profesionales argentinos respecto de su calidad de vida en tanto actores del sistema de salud. Como resultado de encuestas realizadas a médicos, se señala que uno de los factores más preocupantes es el tiempo de duración de la consulta médica. En este sentido, la Sociedad Argentina de Cardiología realizó una amplia encuesta cuyos resultados demostraron que al 70\% de los profesionales se les exige ofrecer turnos de 10 a 15 min, en tanto que ellos pensaban que lo correcto sería que las consultas tuvieran una duración entre 20 y $30 \mathrm{~min}$. Al mismo tiempo consideraron que constituye una falta a la ética aceptar atender pacientes en un tiempo inadecuado ${ }^{14}$.

\section{Planteamiento del problema}

Podríamos decir que, en términos generales, la bibliografía mundial respalda la asignación de pacientes entre 10 y $15 \mathrm{~min}$, tal como ocurre en nuestro medio. Ahora bien, ies este tiempo suficiente? ¿Nos garantiza una buena calidad de atención médica?

Normalmente el primer contacto con el médico generalista implica presentarse al paciente, establecer empatía, confeccionar una buena historia clínica donde se registre la edad, enfermedades crónicas, antecedentes familiares, cumplimiento del plan de vacunación; también debe hacerse un examen semiológico que, entre otras cosas, incluye el control de la tensión arterial, la auscultación cardiopulmonar, la palpación abdominal y la evaluación neurológica; asimismo, la consulta incluye la indicación de exámenes complementarios, edu- 
cación y orientación al paciente. A nivel general, el consenso indica que una CMA menor a 10 min es inaceptable, porque supone riesgos tanto para el paciente (por ej. que no se detecten condiciones patológicas) como para el médico (aumento de incidencia de síndrome de burn out $)^{8,14}$.

Otro factor importante que puede prolongar el tiempo de las CMA es que los pacientes hacen cada vez más preguntas pues tienen mayor acceso a la información y esperan participar más activamente en la toma de decisiones. Esto se corresponde con un cambio de paradigma en el modelo de relación médico paciente, que viene produciéndose desde la irrupción de la bioética en la década de 1970. De un modelo basado en la beneficencia y con un paciente más pasivo, se ha avanzado hacia un modelo basado en la autonomía, con pacientes que participan activamente en busca de lo que consideran lo mejor para sus vidas ${ }^{15}$.

Por otro lado, el incremento en los últimos años de la expectativa de vida -producto de los avances científicos y tecnológicos- hace que los médicos vean cada vez pacientes más añosos y con múltiples enfermedades ${ }^{16}$. Los médicos del nuevo milenio se encuentran en un punto de inflexión donde hay que buscar nuevas alternativas de atención médica que garanticen la calidad en la atención a la vez que se incrementa el número de pacientes asistidos ${ }^{17,18}$.

Finalmente, el escaso tiempo de la consulta puede deteriorar la relación médico paciente (RMP). Desde la Antigüedad se reconoce que la RMP es un factor de enorme importancia para la recuperación de los enfermos ${ }^{19,20}$. Sin duda, es el primer y principal acto médico. Pero, hoy día, el abuso de la tecnología y la tendencia a la despersonalización atentan contra este recurso terapéutico.

Por su parte, la atención primaria de la salud es el primer nivel de atención profesional en la mayoría de los países. Es el nivel donde las personas presentan sus problemas de salud y donde la mayoría de las necesidades en salud encuentran la cura o la prevención. La coordinación del acceso y utilización de estos servicios es uno de los pilares que contribuye a mantener el estado de salud de la población ${ }^{15,16}$.

Un estudio en Alemania demostró la obtención de grandes beneficios en el devenir de pacientes con enfermedades crónicas, donde las consultas médicas tenían un mínimo de $30 \mathrm{~min}$. Parte de estos resultados se asocian con el mayor tiempo para dar indicaciones para mejorar hábitos de vida y establecer un compromiso en la relación médico-paciente, para el cumplimiento de los planes terapéuticos ${ }^{17}$.

Otro factor a tener en cuenta es la forma de remuneración o el tipo de contrato del médico con las instituciones. Por un lado están los contratos per cápita, donde al médico se le paga por cada paciente atendido; en este caso la duración de las consultas puede verse afectada dado que cuantos más pacientes se atiendan más dinero recibirá el médico; esto implica el riesgo de una disminución en los tiempos de consulta como se evidencia en el estudio realizado en Canadá ${ }^{10}$. En los casos de médicos con remuneración fija, la duración de las consultas parece estar más controlada y menos manipulada por parte del profesional ${ }^{18}$.

\section{El tiempo de consulta médica y la calidad de la atención médica}

La OMS define la calidad de manera muy amplia: "la calidad en asistencia sanitaria es asegurar que cada paciente reciba el conjunto de medios diagnósticos y terapéuticos más adecuado para conseguir una atención sanitaria óptima teniendo en cuenta todos los factores y conocimientos del paciente y del servicio médico y lograr el mínimo riesgo de efectos adversos y la máxima satisfacción del paciente con el proceso" 21 . Tiene en cuenta las siguientes variables: alto nivel de excelencia profesional, uso eficiente de los recursos, mínimos riesgos para el paciente, resultados finales en la salud, alto grado de satisfacción por parte del paciente. Puede observarse que en esta definición se consideran tres criterios:

1) criterio técnico (uso adecuado de la ciencia y de la tecnología, con maximización de los beneficios y minimización de los riesgos; primun non nocere o principio de no-maleficencia en el discurso bioético actual)

2) criterio interpersonal (interacción social y psicológica de los actores de la relación clínica)

3) criterio económico (distribución y utilización racional de los recursos)

En otras palabras: no sólo hay que hacer lo correcto sino que hay que "hacer lo correcto correctamente"22.

En los últimos años se ha producido un cambio 
importante en la organización de los sistemas de salud de la mayoría de los países, involucrando la calidad de la atención médica como uno de los pilares fundamentales en la prestación de los servicios de salud. Dentro de los aspectos que se vinculan con la calidad en la atención médica se encuentran la oportunidad, agilidad, accesibilidad, continuidad, suficiencia, seguridad, integridad, racionalidad, eficiencia, humanidad, información, transparencia, consentimiento y grado de satisfacción de los usuarios del sistema ${ }^{23}$. El tiempo de consulta se enmarca dentro de la suficiencia, la integridad y la satisfacción de los usuarios. Desde el punto de vista de calidad, los usuarios del sistema son tanto los pacientes como los profesionales de la salud.

El Colegio Médico Americano recomienda incrementar los esfuerzos para mejorar la calidad de atención enfocándose en la preservación de la relación médico paciente. Reconoce que el tiempo dedicado a la consulta es uno de los elementos de mayor calidad en la atención médica y una condición necesaria para el desarrollo de una RMP adecuada $^{24}$.

También es importante analizar la cantidad de tiempo vs la calidad de tiempo. Esto implica una optimización del tiempo dedicado a la consulta médica evitando interrupciones que afectan la atención dedicada al enfermo, preguntas sin relevancia y desviaciones de la conversación hacia temas no vinculados con el problema médico. La mayoría de los estudios que abordan el tema de la cantidad de tiempo adecuada para una consulta indican que la misma debe oscilar entre 18 y $20 \mathrm{~min}^{24}$.

Asimismo, el tiempo de consulta es uno de los aspectos que marcan el grado de satisfacción, tanto de los pacientes como de los profesionales. Una disminución en el tiempo disminuye la satisfacción de los usuarios, reduce los alcances de la prevención médica, condiciona a una mala prescripción terapéutica e incrementa el riesgo de mala praxis ${ }^{25}$. Varios estudios sugieren que las consultas deberían tener una duración relativa de 20 min para satisfacer a los usuarios y cumplir con los estándares de calidad asistencial2-5.

Una encuesta realizada en México entre los usuarios de los consultorios externos de varios centros asistenciales públicos y privados mostró que "para los usuarios de la seguridad social, los principales elementos que definen la mala calidad de atención son los largos tiempos de espera, los tiempos cortos dedicados a la consulta y la deficiencia en las acciones de revisión y diagnóstico, mientras que para la población abierta dichos elementos están constituidos por el tiempo corto que duran las consultas, las consultas diferidas y el mal trato por el personal que presta los servi$\operatorname{cios}{ }^{26}$. En otro estudio semejante, los principales factores asociados a una percepción general de baja calidad en la atención de las instituciones de salud fueron: el tipo de institución (público o privado), el tiempo de espera para acceder a la consulta, el grado de mejoría del estado de salud luego de la consulta y una duración de la consulta inferior a $20 \mathrm{~min}^{27}$. En este mal trato para los pacientes estaban involucrados, en primer lugar, el personal de recepción y confección de la ficha de ingreso, en segundo lugar el personal que proporciona informes y en tercer lugar el personal médico. La percepción de mala calidad era directamente proporcional al nivel de ingresos y al nivel de instrucción, al tiempo que el nivel de instrucción se relacionaba directamente con el nivel de ingresos. Es de hacer notar que la mayor insatisfacción se refería al tiempo de espera, superado ampliamente (más de dos horas) respecto a lo establecido por la Norma Oficial Mexicana, que considera como bueno un tiempo de espera menor de 30 min.

Este enfoque de evaluación de resultados puede completarse con encuestas que evalúen el grado de satisfacción de los profesionales de la salud que intervienen directamente en la atención médica. En una encuesta llevada a cabo por la Asociación Argentina de Cardiología se puso de manifiesto la creciente frustración en los médicos por la disociación que existe entre sus intentos por brindar una atención ideal y las restricciones que impone el sistema de atención médica con turnos que rondan los 10 ó 15 min para una especialidad con alteraciones de gran prevalencia en la población, donde el control clínico y las indicaciones preventivas son de vital importancia y consumen un tiempo importante ${ }^{14}$.

Finalmente, cuando el análisis de la calidad pasa por los costos, un poco más de tiempo dedicado a las consultas ha demostrado hacer una gran diferencia en términos de mala praxis. De esta manera se asume que el costo extra que, aparentemente, pudieran representar unos minutos más del tiempo profesional es, por lejos, mucho 
Tiempo de consulta ambulatoria - D. Outomuro et al

menor que el costo de posibles transgresiones médico legales ${ }^{28}$.

\section{Consideraciones finales}

De acuerdo a la bibliografía consultada, el promedio de duración de la consulta médica oscila actualmente entre 10 y $15 \mathrm{~min}$, tiempo en apariencia insuficiente. Sin embargo, es el tiempo establecido en nuestro medio, tanto en establecimientos públicos como privados. Tampoco hemos encontrado ninguna recomendación escrita por parte de organismos estatales, que prescriba destinar más tiempo a las consultas de primera vez en las cuales, como es sabido, una anamnesis y examen físico exhaustivos son necesarios para un adecuado diagnóstico. Al respecto, es interesante señalar que sólo alguna bibliografía proveniente de Europa propone destinar $60 \mathrm{~min}$ para la primera consulta de clínica médica y $20 \mathrm{~min}$ en las siguientes consultas ${ }^{1}$.

En conclusión, los administradores de los sistemas de salud deberían repensar la forma en que se organiza la agenda de turnos médicos. Los indicadores habituales, generalmente de índole económica, son insuficientes en una materia tan delicada como es la atención de la salud. Razones de naturaleza médica y fundamentalmente bioéticas, reseñadas en el apartado sobre calidad de atención médica, sugieren la asignación de un tiempo de consulta en clínica médica que supere los 15 min habituales, proponiendo un tiempo éticamente aceptable de $20 \mathrm{~min}$. Entendemos que un tiempo menor a 20 min no permite cumplimentar adecuadamente el acto médico, tanto técnica como humanamente.

\section{Referencias}

1. Errasti F. El hospital como empresa de servicios. En: Errasti F. Principios de gestión sanitaria. Madrid: Ed. Díaz de Santos, S. A; 1997. Pp. 81-101.

2. De la Calle JL, Abejón D, Cid J, del Pozo C, Insausti J, López E, et al. Estándares de calidad asistencial y catálogo de procedimientos de las unidades de dolor crónico. Revista de la Sociedad Española del Dolor 2010; 17 (2): 114-33.

3. Campbell JL, Howie JGR. Changes resulting from increasing appointment lenght: practical and theoretical issues. Br J Gen Pract 1992; 42: 276-8.
4. Landau DA, Bachner YG, Elishkewitz K, Goldstein L, Barneboim E. Patient's views on optimal visit length in primary care. J Med Pract Manage 2007; 23 (1): 12-5.

5. Migongo AW, Charnigo R, Love M, Kryscio R, Fleming ST, Pearce KA. Factors relating to patient visit time with a physician. Med. Decis. Making 2012; 32: 93-104.

6. Wilson A, McDonald P, Hayes L, Cooney J. Health promotion in the general practice consultation: a minute makes a difference. BMJ 1992; 304: 227-30.

7. Astupiña García ES. Tiempo de consulta en medicina general. Hallado en: http://www.medbook.es/forum/ topics/tiempo-de-consulta-en-medicina. Acceso el 17 de noviembre de 2011.

8. Chen LM, Farwell WR, Ashish KJ. Health Care reform: primary care visit duration and quality. Arch Intern Med 2009; 169 (20): 1866-72.

9. Wooldridge AN, Arató N, Sen A, Amenomori M, Fetters D. Truth or fallacy? Three hour wait for three minutes with the doctor: Findings from a private clinic in rural Japan. Asia Pacific Family Medicine 2010; 9: 11-8.

10. Zaher Zaghloul AA, Abou El Enein NY. Hourly-block and standard patient scheduling systems at two private hospitals in Alexandria. J Multidisc Healthcare 2010, 3 : 225-32.

11. Kringos DS, Boerma WGW, Spaan E, Pellny M. A snapshot of the organization and provision of primary care in Turkey. BMC Health Serv Res 2011; 11: 90-7.

12. Birhanu Z, Assefa T, Woldie M, Morankar S. Determinants of satisfaction with health care provider interactions at health centres in central Ethiopia: a cross sectional study. BMC Health Serv Res 2010; 10: 78-89.

13. Geneau R, Lehoux P, Poneault R, Lamarche P. Understanding the work of general practitioners: a social science perspective on the context of medical decision making in primary care. BMC Family Practice 2008; 9: 12-21.

14. Doval HC. Malestar en la medicina. Insatisfacción y descontento en los médicos. Rev Arg Cardiol 2007; 75 (4): 336-9.

15. Boerma WGW, Dubois CA: Mapping primary care across Europe. En: Primary care in the driver's seat? Organizational reform in European primary care. Eds Saltman RB, Rico A, Boerma WGW. Berkshire: Open University Press; 2006. Pp. 22-49.

16. Kringos DS, Boerma WGW, Bourgueil Y, Cartier T, Hasvold T, Hutchinson A, et al. The european primary care monitor: structure, process and outcome indicators. BMC Fam Pract 2010, 11: 81-8.

17. Hamre HJ, Witt CM, Glockmann A, Ziegler R, Willich $\mathrm{SN}$, Kiene H. Anthroposophic medical therapy in chronic disease: a four-year prospective cohort study. BMC 
Complementary and Alternative Medicine 2007; 7: 10 21.

18. Van den Berg MJ, de Bakker DH, Westert GP, van der Zee J, Groenewegen PP. Do list size and remuneration affect GPs' decisions about how they provide consultations? BMC Health Services Research 2009; 9: 39-49.

19. Kaba R, Sooriakumaran P. The evolution of the doctorpatient relationship. Int. J Surg 2007; 5 (1): 57-65.

20. Fornaro M, Clementi N, Fornaro P. Medicine and psychiatry in Western culture: Ancient Greek myths and Modern prejudices. Ann Gen Psychiat 2009; 8 (1): 21-8.

21. Lemus JD, Aragües y $\mathrm{Oroz}$ V. Auditoría médica. Un enfoque sistémico. Buenos Aires: Ed. Universitarias; 2004.

22. Lemus JD. Epidemiología en Salud Pública y Administración de Servicios de Salud. Buenos Aires, Ed. La Librería de la Ciencia, 2004.

23. Malagón-Londoño G, Galán Morera R, Pontón Laverde G. Administración hospitalaria. Bogotá: Editorial Panamericana; 1996.

24. Braddock CH, Snyder L. The doctor will see you shortly.
The ethical significance of time for the patient-physician relationship. J Gen Intern Med 2005; 20: 1057-62.

25. Lin CT, Albertson GA, Schilling LM, Cyran EM, Anderson $\mathrm{SN}$, Ware $\mathrm{L}$, et al. Is patients' perception of time spent with the physician a determinant of ambulatory patient satisfaction? Arch Intern Med 2001; 161: 143742.

26. Ortiz Espinosa RM, Muñoz Juárez S, Martín del Campo DL, Torres Carreño E. Consulta externa en instituciones de Salud de Hidalgo, México, según la opinión de los usuarios. Pan Am J Public Health 2003; 13 (4): 229-38.

27. Sauceda-Valenzuela AL, Wirtz VJ, Santa-Ana-Tellez Y, Kageyama-Escobar ML. Ambulatory health service users' experience of waiting time and expenditure and factors associated with the perception of low quality of care in Mexico. BMC Health Services Research 2010; 10 : 178-188.

28. Hansson MG, Kihlbom U, Tuvenmo T, Olsen LA, Rodríguez A. Ethics takes time, but not that long. BMC Medical Ethics 2007; 8: 6-12. 